\title{
Two-phase flow of dusty fluid with suspended hybrid nanoparticles over a stretching cylinder with modified Fourier heat flux
}

\author{
R. S. Varun Kumar ${ }^{1}$ R. J. Punith Gowda $\cdot$ R. Naveen Kumar ${ }^{1}$ 'M. Radhika ${ }^{2} \cdot$ B. C. Prasannakumara ${ }^{1}$ (i)
}

Received: 12 November 2020 / Accepted: 10 February 2021 / Published online: 27 February 2021

(c) The Author(s) $2021 \quad$ OPEN

\begin{abstract}
The current paper explores the influence of hybrid nanoparticles on the dusty liquid flow through a stretching cylinder by employing the modified Fourier heat flux law. Two phase model is implemented in the present research to characterise the fluid flow. Molybdenum disulphide and silver are used as nanoparticles suspended in base fluid water. The equations which represent the described flow are changed into a set of ordinary differential equations by opting appropriate similarity variables. The reduced dimensionless nonlinear ODEs are numerically solved out by using Runge-Kutta-Fehlberg fourth fifth order inclusive of shooting approach. The impact of several dimensionless parameters over velocity and thermal gradients are deliberated by using graphs. Graphical illustrations for skin friction are also executed. Result outcome reveals that, rise in values of mass concentration of particle declines the velocity and thermal gradient of both dust and fluid phases and cumulative in curvature parameter upsurges the velocity and thermal gradient within the boundary. Further, the heightening of thermal relaxation parameter enhances the thermal profile of both fluid and dust phases.
\end{abstract}

Keywords Dusty hybrid nanofluid $\cdot$ Stretching cylinder $\cdot$ Mixed convection $\cdot$ Cattaneo-Christov heat flux

\section{List of symbols}

$(u, v)\left(u_{p}, v_{p}\right) \quad$ Components of velocity

$(x, r)$

$f^{\prime}(\eta)$

$\Theta$

$\mu$

$\mathrm{F}^{\prime}(\eta)$

$\rho$

$\mathrm{k}$

$\left(\rho C_{p}\right)$

I

$\mathrm{K}$

$\mathrm{T}$

$\mathrm{C}_{\mathrm{f}}$

$\tau_{\mathrm{t}}$

$\mathrm{N}$

$f$

L
Directions

Dimensionless Fluid velocity

Non-dimensional fluid phase temperature

Dynamic viscosity

Dimensionless velocity of dust particle

Density

Thermal conductivity

Heat capacitance

Mass concentration of particles

Stokes drag constant

Temperature

Skin friction coefficient

Thermal equilibrium time

Dust particle density number

Fluid

Reference length gr Mixed convection parameter

a Stretching rate

$\beta_{\mathrm{t}}$ and $\beta_{\mathrm{v}} \quad$ Interaction parameters of temperature and velocity of the fluid

Kinematic viscosity

Mass of the dust particles

$\begin{array}{ll}\mathrm{m} & \text { Mass of the dust } \\ \mathrm{Gr} & \text { Greshof number }\end{array}$

$\tau_{\mathrm{v}}=\frac{\mathrm{m}}{\mathrm{k}} \quad$ Relaxation time of dust particle

$\mathrm{C}$

$\mathrm{Re}_{\mathrm{x}} \quad$ Local Reynolds number

$\Gamma \quad$ Thermal relaxation parameter

Ec Eckert number

$\phi_{1}, \phi_{2} \quad$ Volume fractions

$\delta \quad$ Curvature parameter

$\mathrm{Pr} \quad$ Prandtl number

$\gamma^{*} \quad$ Relaxation time

$\mathrm{U}_{\mathrm{w}}(\mathrm{x}) \quad$ Stretching velocity

$\mathrm{Bi} \quad$ Biot number

B. C. Prasannakumara, dr.bcprasanna@gmail.com | 'Department of Studies and Research in Mathematics, Davangere University, Davangere, Karnataka, India. ${ }^{2}$ Government First Grade College, Kolar Gold Field, Karnataka 563122, India. 


$\begin{array}{ll}\mathrm{R} & \text { Radius of the cylinder } \\ \mathrm{bf} & \text { Base fluid } \\ \mathrm{p} & \text { Dust particles } \\ \mathrm{hnf} & \text { Hybrid nanofluid } \\ \infty & \text { Ambient } \\ \mathrm{w} & \text { Surface } \\ \mathrm{s}_{1}, \mathrm{~s}_{2} & \text { Solid particles }\end{array}$

\section{Introduction}

From the past decades, scientists were involved in performing a noteworthy investigation in the fluid flow area with respect to heat transfer process. Invention of special fluids paved a way for obtaining higher efficient energy during heat transfer. Researchers used several techniques and methods to improve efficiency of fluids. These fluids are termed as "nanofluids", which are prepared by dispersing minute particles of size less than $100 \mathrm{~nm}$ in the conventional fluids. In modern science and nanotechnology, the rheological performance of a nanofluid is an essential feature for many applications such as cooling process, Geothermal power extraction, Coolant, Lubrication in automobiles and Microchips cooling. Therefore, significant studies have been performing on these nanofluids. Hayat et al. [1] deliberated the nano liquid flow at stagnation point with Brownian motion. They discussed the demeanour of physical parameters versus different profiles via graphical representation. Flow of a fluid consisting of nanoparticles through a revolving disk was explained by Khan et al. [2]. Kuttan et al. [3] deliberated the performance of four diverse nanoparticles on boundary layer stream through a stretching surface. Vinita and Poply [4] examined the impact of heat generation on nanofluid flow past a stretching cylinder. Mondal et al. [5] examined the impact of viscous dissipation and mixed convection on nanoliquid stream past a stretching cylinder. The combination of dual or more nanoparticles which are suspended in a convectional liquid yield's hybrid nanofluid. Enrichment of heat transfer in hybrid nanofluid is mainly caused by fusion of two or more nanoparticles. It is experimentally verified that, hybrid nanofluids offer better thermal behaviour than that of base fluids and single nanoparticle fluids. Recently, Khan et al. [6] considered a fluid flow of consisting two nanometallic substances namely, silicon dioxide and molybdenum disulphide for determining the impact of heat source or sink. Farooq et al. [7] explored optimization of entropy on the flow of nanohybrid fluid through a revolving channel. Manjunatha et al. [8] examined the impact of convective boundary constraints on micropolar hybrid nanoliquid stream past a stretching surface. Abbas et al. [9] deliberated the stagnation point stream of a hybrid nanoliquid past a stretching cylinder.
The fluid flows along with dust particles have extensive range of mechanical applications like transport processes, cement and steel manufacturing industries, flying ash from thermal plants and chilling consequence of $A C$ 's. The two-phase flows involving solid particles scattered in a nanofluid and hybrid nanofluid are of significant applications in industries. Recently, several researchers scrutinised the fluid flow with dust particles suspension. Turkyilmazoglu [10] provided a mathematical explanation for dusty fluid flow along with the boundary conditions. Manjunatha et al. [11] illustrated the dusty fluid flow through a stretchy cylinder on taking account of heat source. Reddy et al. [12] imposed modified Fourier law to investigate dusty hybrid nanofluid flow over a stretching surface with porous medium. Radhika et al. [13] constructed two-way flow approaching model to investigate energy transport in hybrid dusty nanofluid. Mallikarjuna et al. [14] examined effect of viscous dissipation on dusty hybrid nanoliquid stream past a stretching surface. Abdelmalek et al. [15] discussed the heat transference in dusty hybrid nano liquid stream.

The flow of different fluids over a cylinder has gained special interest in research area with many significant uses such as revolving-tube heat exchangers, cooling of electronic apparatus, and drilling of oil wells. Hayat et al. [16] used a stretched cylinder to evaluate mixed convective stream of liquid with appropriate boundary conditions. Khan et al. [17] deliberated the hydromagnetic viscous liquid flow through a cylinder. Waqas et al. [18] inspected the influence of activation energy on the nanoparticle fluid flow generated by a cylinder. The heat transfer in MHD nanofluid stream on taking account of ohmic heating over a stretching cylinder was deliberated by Mishra and Kumar [19]. Mixed convective flow and heat transfer process has enormous engineering applications like, solar cells, drying, electronic cooling devices and many others. The scrutiny of mixed convection is complex due to the convoluted interaction between the free convection with the shear motivated flow. Qayyum et al. [20] studied mixed convective two-dimensional nanofluid flow at a stagnation point over a sheet. The entropy optimization of Eyring Powell liquid flow with nanoparticles was analysed by Alsaedi et al. [21]. Further mixed convective heat transfer is scrutinized here. Akbar et al. [22] schematically illustrated mixed convective stream of nanofluid with various slip conditions. Ramesh et al. [23] deliberated the mixed convective stream of hybrid nanofluid past an upright permeable cylinder.

Fourier's law has been practically applied for studying heat transfer problems. But the most important complexity of this law is that it yields a parabolic equation of energy, which specifies that primary disturbance has established all over the entire system. To beat this difficulty, Cattaneo modified this law with the help of heat flux relaxation 
time. Later on, Christov improved Cattaneo's proposition with suitable explanation. Further, many authors used the modified Fourier model in their studies. Salahuddin et al. [24] examined the impact of modified Fourier heat flux on Williamson liquid past a stretching sheet. Modified Fourier model was implemented by Khan et al. [25] to estimate the hydromagnetic flow of Carreau liquid through a cylinder. Hussain et al. [26] examined the heat transfer features in viscoinelastic liquid past a stretching surface by means of modified Fourier heat flux. Ahmed et al. [27] scrutinised the mixed convective stream of maxwell liquid on taking account of Catteneo-Christov heat flux. Khan et al. [28] deliberated the impact of modified Fourier heat flux on non-Newtonian liquid stream past a stretching cylinder.

In view of the above literature survey, it is obvious to the best of writer's knowledge, that no efforts have far been initiated with respect to modified Fourier heat flux effect on 2D-stream of a dusty hybrid nanoliquid over a stretching cylinder with mixed convection which constitutes the novelty of the present work. In this study, we discuss the consequence of hybrid nanoparticles on the dusty fluid flow through a stretching cylinder by utilising the modified Fourier heat flux law. The dusty-fluid expressions for dust and nanoparticles are discussed by using equations [1-6], and are employed with corresponding boundary conditions [7], both the dust and fluid particles are governed by the coupled set of the energy and momentum expressions. Further, we chose Molybdenum disulphide and silver as nanoparticles suspended in carrier liquid water. The governing coupled non-linear PDEs are numerically solved and the outcomes attained are presented graphically and discussed briefly. The solutions presented are not only important for practical applications but also work for further research on an ongoing topic that incorporates more complex physical factors, as quoted in the aforementioned articles in this field.

The structure of the paper consists of five parts: Sect. 1 summarizes a few published investigations and the motives for this investigation; Sect. 2 reviews the methodology used; Sect. 3 describes the Numerical method opted: the outcomes of this study and discussion are presented in Sect. 4; the conclusions and recommendations are given in the last section.

\section{Mathematical formulation}

Consider a two-dimensional incompressible dusty hybrid nanofluid flow through a stretching cylinder with reference length $L$ and stretching ratea. The stretching velocity is denoted by $U_{w}(x)$ which is given by $U_{w}(x)=\frac{a x}{L}$. The axis of the cylinder is treated along the $x$-axis and radial direction along the $r$ axis. The analysis of heat transfer is done by considering modified Fourier heat flux and mixed convection. The equations which represent the hybrid nanofluid flow with dust particle suspension on taking account of mixed convection through a stretching cylinder is given by (refer Manjunatha et al. [11]):

\subsection{Fluid phase}

$$
\frac{\partial(\mathrm{ru})}{\partial \mathrm{x}}+\frac{\partial(\mathrm{rv})}{\partial \mathrm{r}}=0
$$

$$
\begin{gathered}
\left(u \frac{\partial u}{\partial x}+v \frac{\partial u}{\partial r}\right)=\frac{\mu_{h n f}}{\rho_{h n f}}\left(\frac{\partial^{2} u}{\partial r^{2}}+\frac{1}{r} \frac{\partial u}{\partial r}\right) \\
+\frac{K N}{\rho_{h n f}}\left(u_{p}-u\right)+\frac{g(\rho \beta)_{h n f}\left(T-T_{\infty}\right)}{\rho_{h n f}}
\end{gathered}
$$

$$
\begin{aligned}
& \left(\mathrm{u} \frac{\partial \mathrm{T}}{\partial \mathrm{x}}+v \frac{\partial \mathrm{T}}{\partial \mathrm{r}}\right)+\gamma^{*}\left(u^{2} \frac{\partial^{2} \mathrm{~T}}{\partial \mathrm{x}^{2}}+v^{2} \frac{\partial^{2} \mathrm{~T}}{\partial \mathrm{r}^{2}}+2 \mathrm{uv} \frac{\partial^{2} \mathrm{~T}}{\partial \mathrm{r} \partial \mathrm{x}}\right. \\
& \left.+\mathrm{u} \frac{\partial \mathrm{u}}{\partial \mathrm{r}} \frac{\partial \mathrm{T}}{\partial \mathrm{x}}+u \frac{\partial \mathrm{v}}{\partial \mathrm{x}} \frac{\partial \mathrm{T}}{\partial \mathrm{r}}+v \frac{\partial \mathrm{u}}{\partial \mathrm{x}} \frac{\partial \mathrm{T}}{\partial \mathrm{x}}+v \frac{\partial \mathrm{v}}{\partial \mathrm{r}} \frac{\partial \mathrm{T}}{\partial \mathrm{r}}\right) \\
& =\frac{\mathrm{k}_{\mathrm{hnf}}}{\left(\rho C_{\mathrm{p}}\right)_{\mathrm{hnf}}}\left(\frac{\partial^{2} \mathrm{~T}}{\partial \mathrm{r}^{2}}+\frac{1}{\mathrm{r}} \frac{\partial \mathrm{T}}{\partial \mathrm{r}}\right) \\
& +\frac{\rho_{\mathrm{p}}}{\left(\rho C_{\mathrm{p}}\right)_{\mathrm{hnf}} \tau_{v}}\left(\mathrm{u}_{\mathrm{p}}-\mathrm{u}\right)^{2}+\frac{\rho_{\mathrm{p}}\left(\mathrm{C}_{\mathrm{p}}\right)_{\mathrm{f}}}{\left(\rho C_{\mathrm{p}}\right)_{\mathrm{hnf}} \tau_{t}}\left(\mathrm{~T}_{\mathrm{p}}-\mathrm{T}\right)
\end{aligned}
$$

\subsection{Dust phase}

$\frac{\partial\left(\mathrm{ru}_{\mathrm{p}}\right)}{\partial \mathrm{x}}+\frac{\partial\left(\mathrm{rv}_{\mathrm{P}}\right)}{\partial \mathrm{r}}=0$

$\left(u_{p} \frac{\partial u_{p}}{\partial x}+v_{p} \frac{\partial u_{p}}{\partial r}\right)=\frac{K}{m}\left(u-u_{p}\right)$

$\left(u_{p} \frac{\partial T_{p}}{\partial x}+v_{p} \frac{\partial T_{p}}{\partial r}\right)=-\frac{C_{p f}}{C_{m} \tau_{T}}\left(T_{p}-T\right)$

Boundary constraints for the proposed problem are:

$$
\begin{gathered}
\mathrm{u}=\mathrm{U}_{\mathrm{w}}, \mathrm{v}=0,-\mathrm{k}_{\text {hnf }} \frac{\partial \mathrm{T}}{\partial \mathrm{x}}=\Omega_{1}\left(\mathrm{~T}_{\mathrm{w}}-\mathrm{T}\right) \text { at } \mathrm{r}=\mathrm{R} \\
\mathrm{u} \rightarrow 0, \mathrm{u}_{\mathrm{p}} \rightarrow 0, \mathrm{v}_{\mathrm{p}} \rightarrow \mathrm{v}, \mathrm{T} \rightarrow \mathrm{T}_{\infty}, \mathrm{T}_{p} \rightarrow \mathrm{T}_{\infty} \text { as } \mathrm{r} \rightarrow \infty
\end{gathered}
$$

The suitable similarity variables are:

$u=\frac{a x}{L} f^{\prime}(\eta), v=-\frac{1}{r} \sqrt{\frac{v_{f} a}{L}} R f(\eta), \eta=\frac{r^{2}-R^{2}}{2 R} \sqrt{\frac{a}{v_{f} L}}, u_{p}=\frac{a x}{L} F^{\prime}(\eta)$, $v_{p}=-\frac{1}{r} \sqrt{\frac{v_{f} \mathrm{a}}{L}} R F(\eta) \theta(\eta)=\frac{T-T_{\infty}}{T_{w}-T_{\infty}}, \theta_{p}(\eta)=\frac{T_{p}-T_{\infty}}{T_{w}-T_{\infty}}$

where $\rho_{\text {hnf }} \mu_{\text {hnf }}\left(\rho C_{p}\right)_{\text {hnf }}(\rho \beta)_{\text {hnf }}$ and $k_{\text {hnf }}$ is defined by: 


$$
\begin{aligned}
& \frac{\rho_{\mathrm{hnf}}}{\rho_{\mathrm{f}}}=\left(1-\phi_{2}\right)\left[\left(1-\phi_{1}\right)+\phi_{1} \frac{\rho_{\mathrm{s} 1}}{\rho_{\mathrm{f}}}\right]+\phi_{2} \frac{\rho_{\mathrm{s} 2}}{\rho_{\mathrm{f}}} \\
& \frac{(\rho \beta)_{\mathrm{hnf}}}{(\rho \beta)_{\mathrm{f}}}=\left(1-\phi_{2}\right)\left[\left(1-\phi_{1}\right)+\phi_{1} \frac{(\rho \beta)_{s 1}}{(\rho \beta)_{\mathrm{f}}}\right]+\phi_{2} \frac{(\rho \beta)_{s 2}}{(\rho \beta)_{\mathrm{f}}} \\
& \frac{(\rho C \rho)_{\mathrm{hnf}}}{\left(C_{\mathrm{p}} \rho\right)_{\mathrm{f}}}=\left(1-\phi_{2}\right)\left[\left(1-\phi_{1}\right)+\phi_{1}\left(\frac{(\rho C p)_{s 1}}{(\rho C p)_{\mathrm{f}}}\right)\right]+\phi_{2} \frac{(\rho C p)_{s 2}}{(\rho C p)_{\mathrm{f}}} \\
& \mu_{\mathrm{hnf}}=\frac{\mu_{\mathrm{f}}}{\left(1-\phi_{1}\right)^{2.5}\left(1-\phi_{2}\right)^{2.5}} \\
& \frac{\mathrm{k}_{\mathrm{hnf}}}{\mathrm{k}_{\mathrm{bf}}}=\frac{\mathrm{k}_{\mathrm{s}_{2}}+2 \mathrm{k}_{\mathrm{bf}}-2 \varphi_{2}\left(\mathrm{k}_{\mathrm{bf}}-\mathrm{k}_{\mathrm{s}_{2}}\right)}{\mathrm{k}_{\mathrm{s}_{2}}+2 \mathrm{k}_{\mathrm{bf}}+\varphi_{2}\left(\mathrm{k}_{\mathrm{bf}}-\mathrm{k}_{\mathrm{s}_{2}}\right)}, \frac{\mathrm{k}_{\mathrm{bf}}}{\mathrm{k}_{\mathrm{f}}}=\frac{\mathrm{k}_{\mathrm{s}_{1}}+2 \mathrm{k}_{\mathrm{f}}-2 \varphi_{1}\left(\mathrm{k}_{\mathrm{f}}-\mathrm{k}_{s_{1}}\right)}{\mathrm{k}_{\mathrm{s}_{1}}+2 \mathrm{k}_{\mathrm{f}}+\varphi_{1}\left(\mathrm{k}_{\mathrm{f}}-\mathrm{k}_{\mathrm{s}_{1}}\right)}
\end{aligned}
$$

By using above similarity transformation, the obtained reduced equations are as follow:

$\varepsilon_{1}\left[(2 \eta \delta+1) f^{\prime \prime}+2 \delta f^{\prime \prime}\right]-\left[f^{\prime 2}-f f^{\prime \prime}\right]+\varepsilon_{2} I \beta_{v}\left(F^{\prime}-f^{\prime}\right)+\frac{\varepsilon_{2}}{\varepsilon_{3}} g r \theta=0$

$$
\begin{aligned}
& \varepsilon_{4}\left(\frac{\mathrm{k}_{\mathrm{hnf}}}{\mathrm{k}_{\mathrm{f}}}\right)\left[\frac{1}{\mathrm{Pr}}\right]\left[(2 \delta \eta+1) \theta^{\prime \prime}+2 \delta \theta^{\prime}\right]+\mathrm{f} \theta^{\prime} \\
& \quad+\varepsilon_{4} / \beta_{v} \operatorname{Ec}\left(\mathrm{F}^{\prime}-\mathrm{f}^{\prime}\right)^{2}+\varepsilon_{4} / \beta_{t}\left(\theta_{p}+\theta^{\prime}\right)-\Gamma\left(f^{2} \theta^{\prime \prime}+f f^{\prime} \theta^{\prime}\right)=0
\end{aligned}
$$

$F F^{\prime \prime}-F^{\prime 2}+\beta_{v}\left(f^{\prime}-F^{\prime}\right)=0$

$\mathrm{F} \theta_{p}^{\prime}-\beta_{t} \gamma\left(\theta_{p}-\theta\right)=0$

where

$$
\begin{aligned}
\varepsilon_{1} & =\frac{1}{\left(1-\phi_{1}\right)^{2.5}\left(1-\phi_{2}\right)^{2.5}\left[\left(1-\phi_{2}\right)\left[\left(1-\phi_{1}\right)+\phi_{1} \frac{\rho_{s 1}}{\rho_{f}}\right]+\phi_{2} \frac{\rho_{s 2}}{\rho_{f}}\right]} \\
\varepsilon_{2} & =\frac{1}{\left(1-\phi_{2}\right)\left[\left(1-\phi_{1}\right)+\phi_{1} \frac{\rho_{s 1}}{\rho_{f}}\right]+\phi_{2} \frac{\rho_{s 2}}{\rho_{f}}} \\
\varepsilon_{3} & =\frac{1}{\left(1-\phi_{2}\right)\left[\left(1-\phi_{1}\right)+\phi_{1} \frac{\rho_{s} 1}{\left(\rho \beta_{f}\right.}\right]+\phi_{2} \frac{\rho_{s 2}}{\left(\rho \beta_{f}\right.}} \\
\varepsilon_{4} & =\frac{1}{\left(1-\phi_{2}\right)\left[\left(1-\phi_{1}\right)+\phi_{1}\left(\frac{(\rho C p)_{s 1}}{\left(\rho C p_{f}\right.}\right)\right]+\phi_{2} \frac{(\rho C p)_{s 2}}{(\rho C p)_{f}}}
\end{aligned}
$$

The corresponding reduced boundary constraints are:

$f(0)=0, f^{\prime}(0)=1, \frac{k_{h n f}}{k_{f}} \theta^{\prime}(0)=-B i(1-\theta(0))$

$f^{\prime}(\infty)=0, F^{\prime}(\infty)=0, F(\infty)-f(\infty)=0, \theta(\infty)=0, \theta_{p}(\infty)=0$

where,

$\beta_{\mathrm{t}}=\frac{\mathrm{L}}{\tau_{\mathrm{t}} a} \beta_{\mathrm{v}}=\frac{\mathrm{L}}{\tau_{\mathrm{v}} a} \delta=\sqrt{\frac{\nu_{\mathrm{f}} \mathrm{L}}{\mathrm{aR}^{2}}} \gamma=\frac{\mathrm{C}_{\mathrm{pf}}}{\mathrm{C}_{\mathrm{m}}}, \operatorname{Pr}=\frac{\left(\rho C_{\mathrm{p}}\right)_{\mathrm{f}} \nu_{\mathrm{f}}}{\mathrm{k}_{\mathrm{f}}}$,

$\operatorname{Re}_{\mathrm{x}}=\frac{U_{\mathrm{w}} \mathrm{x}}{\nu_{\mathrm{f}}}, \mathrm{Gr}=\frac{\mathrm{g}(\beta)_{\mathrm{f}}\left(\mathrm{T}_{\mathrm{w}}-\mathrm{T}_{\infty}\right) \mathrm{x}^{3}}{\nu_{\mathrm{f}}^{2}}, \mathrm{I}=\frac{\mathrm{mN}}{\rho_{\mathrm{f}}}$,

$g r=\frac{\mathrm{Gr}}{\mathrm{Re}_{\mathrm{x}}^{2}}, \Gamma=\frac{\gamma^{*} \mathrm{a}}{\mathrm{L}}, B i=\frac{\Omega_{1}}{\mathrm{k}_{\mathrm{f}}} \sqrt{\frac{\nu_{\mathrm{f}} \mathrm{L}}{a}}$

The skin friction coefficient is given by
$C_{f}=\frac{\mu_{h n f}}{\rho_{f} u_{w}^{2}}\left(\frac{\partial u}{\partial r}\right)_{r=R}$

By using non-dimensional variables, we have

$$
C_{f} \operatorname{Re}_{x} \frac{1}{2}=\frac{f^{\prime \prime}(0)}{\left(1-\phi_{1}\right)^{2.5}\left(1-\phi_{2}\right)^{2.5}}, \text { Where } \operatorname{Re}_{x}=\frac{U_{w} x}{v_{f}}
$$

\section{Numerical solution}

To understand the phenomena of the model, the non-linear differential Eqs. (8-11) with corresponding boundary constraints (12-13) are cracked by applying Runge-Kutta-Fehlberg-45 (RKF-45) technique along with the shooting method. The ordinary differential equations which are coupled, nonlinear with boundary constraints are numerically solved and scrutinized with the help of graphs. To solve these reduced equations, corresponding equations along with boundary conditions are transformed into accompanying arrangement of differential equations in first-order using the replacements $f=y(1), f^{\prime}=y(2), f^{\prime \prime}=y(3), \theta=y(4), \theta^{\prime}=y(5)$, $\mathrm{F}=\mathrm{y}(6), \mathrm{F}^{\prime}=y(7)$, and $\theta_{\mathrm{p}}=\mathrm{y}(8)$.

$\mathrm{y}^{\prime}(1)=\mathrm{y}(2)$

$\mathrm{y}^{\prime}(2)=\mathrm{y}(3)$

$y^{\prime}(3)=\frac{-\varepsilon_{1}[2 \delta y(3)]+\left[y(2)^{2}-y(1) y(3)\right]-\varepsilon_{2} / \beta_{v}(y(7)-y(2))-\frac{\varepsilon_{2}}{\varepsilon_{3}} \operatorname{gr~} y(4)}{\varepsilon_{1}(2 \eta \delta+1)}$

$\mathrm{y}^{\prime}(4)=\mathrm{y}(5)$

$\mathrm{y}(6)=\mathrm{y}(7)$

$y^{\prime}(7)=\frac{y(7)^{2}-\beta_{v}(y(2)-y(7))}{y(6)}$

$y^{\prime}(8)=\frac{\beta_{t} \gamma(y(8)-y(4))}{y(6)}$

The Runge Kutta Fehlberg-45 order algorithm is given by:

$k_{1}=h f\left(t_{k}, y_{k}\right)$

$k_{2}=h f\left(t_{k}+\frac{1}{4} h, y_{k}+\frac{1}{4} k_{1}\right)$

$k_{3}=h f\left(t_{k}+\frac{3}{8} h, y_{k}+\frac{3}{32} k_{1}+\frac{9}{32} k_{2}\right)$

$k_{4}=h f\left(t_{k}+\frac{12}{13} h, y_{k}+\frac{1932}{2197} k_{1}-\frac{7200}{2197} k_{2}+\frac{7296}{2197} k_{3}\right)$

$k_{5}=h f\left(t_{k}+h, y_{k}+\frac{439}{216} k_{1}-8 k_{2}+\frac{3680}{513} k_{3}-\frac{845}{4104} k_{4}\right)$

$k_{6}=h f\left(t_{k}+\frac{1}{2} h, y_{k}-\frac{8}{27} k_{1}+2 k_{2}-\frac{3544}{2565} k_{3}+\frac{1859}{4104} k_{4}-\frac{11}{40} k_{5}\right)$

$z_{k+1}=y_{k}+\frac{16}{135} k_{1}+\frac{6656}{12825} k_{3}+\frac{28561}{56430} k_{4}-\frac{9}{50} k_{5}+\frac{2}{55} k_{6}$

We have selected a distinct value of $\eta_{\infty}$ so that far field boundary constraints are satisfied asymptotically. In this present study, an apt finite value of $\eta_{\infty}$ is considered as $\eta_{\infty}<7$ in such a way that not only numerical solutions converge but also boundary conditions defined at infinity 
Table 1 Comparison of the results for the dimensionless temperature gradient when $\varepsilon_{1}=\frac{\boldsymbol{k}_{\text {hnf }}}{\boldsymbol{k}_{\boldsymbol{f}}}=1$

$\boldsymbol{\delta}=\stackrel{\boldsymbol{K}_{f}}{=} \varepsilon_{2}=\varepsilon_{4}=0$

\begin{tabular}{lllllll}
\hline $\operatorname{Pr}$ & $\begin{array}{l}\text { Grubka and } \\
\text { Bobba [29] }\end{array}$ & $\begin{array}{l}\text { Abel and } \\
\text { Mahesha [30] }\end{array}$ & Ali [31] & Ishak et al. [32] & $\begin{array}{l}\text { Manjunatha } \\
\text { et al. [11] }\end{array}$ & Present work \\
\hline 1.0 & 1.3333 & 1.3333 & 1.3269 & 1.3333 & 1.3333 & 1.3333 \\
10 & 4.7969 & 4.7968 & 4.7969 & 4.7969 & 4.7968 & 4.7969 \\
\hline
\end{tabular}

Table 2 Thermo physical properties of $\boldsymbol{M o S}_{2}, \boldsymbol{A g}$ and water (Refer Shafie et al. [33])

\begin{tabular}{llll}
\hline Physical properties & $\mathrm{MoS}_{2}$ & $\mathrm{Ag}$ & $\mathrm{H}_{2} \mathrm{O}$ \\
\hline$\rho\left(\mathrm{kg} / \mathrm{m}^{3}\right)$ & $5.06 \times 10^{3}$ & 10500 & 997.1 \\
$\mathrm{C}_{p}(\mathrm{~J} / \mathrm{kg} . K)$ & 397.21 & 235 & 4179 \\
$k(W / m . K)$ & 904.4 & 429 & 0.613 \\
$\beta \times 10^{-5}\left(\mathrm{~K}^{-1}\right)$ & 2.8424 & 1.89 & 21 \\
\hline
\end{tabular}

satisfy asymptotically. The tolerance for related errors up to $10^{-8}$ is considered for convergence and the step size is chosen as $\Delta \eta=0.001$. In addition, the CPU time to measure velocity values $(1.34 \mathrm{~s})$ is much shorter than the CPU time to measure temperature values (1.74 s) and the CPU concentration time is $2.01 \mathrm{~s}$. We also compared the numerical results obtained in the present study with corresponding results of the published work. Table 1 is constructed for comparative table of present results with existing work and obtained a good agreement with each other.

\section{Results and discussions}

This segment treats the outcomes of the mixed convective flow of dusty hybrid Nano liquid over a cylinder on taking account of Cattaneo-Christov heat flux. Two phase model is implemented in the present research to characterise the fluid flow. Here, we chosen Molybdenum disulphide and silver as nanoparticles suspended in water as carrier liquid. A numerical scheme is applied to provide a clear understanding and are sufficient to depict the behaviour of flow profiles, which are strategized and deliberated with the assistance of graphs. The thermo-physical characteristics of nanoparticles and base liquid are introduced in Table 2. In this segment, we emphasis on the reliability of non-dimensional parameters that can contribute to velocity and temperature profiles containing dust and hybrid nanoparticles. The choice of the first guess and the extent of the boundary layer $\eta_{\infty}$ depends on the parameters values which are used to find the essential solutions. The values selected in computations are based on associated earlier investigation. The physical parameter values are set and for various values of mass concentration of particles, mixed convection parameter, fluid particles interaction parameter over velocity and thermal gradients of fluid

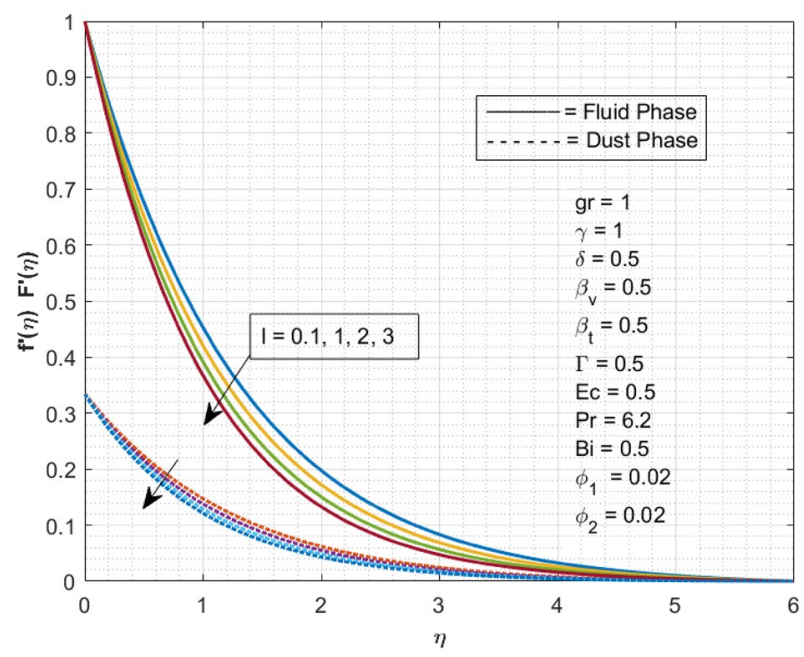

Fig. 1 Power of $I$ over $f^{\prime}(\eta), F^{\prime}(\eta)$

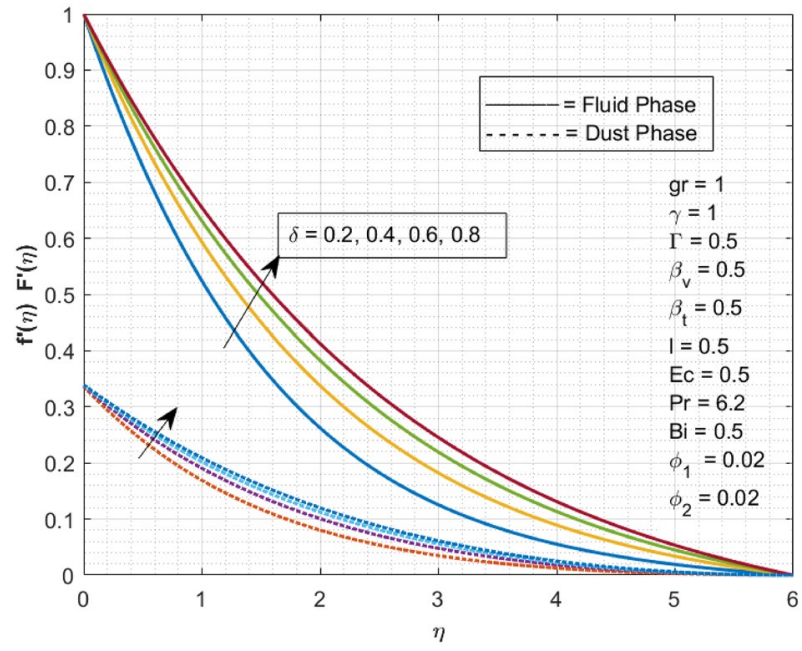

Fig. 2 Power of $\delta$ over $f^{\prime}(\eta), F^{\prime}(\eta)$

containing dust particles and hybrid nanoparticles. Further, they are exemplified with the help of graphs.

Figure 1 is plotted to see the domination of $/$ over velocity gradient of both fluid and dust phases. The upshot in values of I deteriorates the velocity of both phases as depicted in Fig. 1. It is perceptible that the upsurge in dust particles mass concentration diminishes fluid velocity and related boundary layer thickness. The sway of curvature parameter on velocity gradient of both phases is put on 


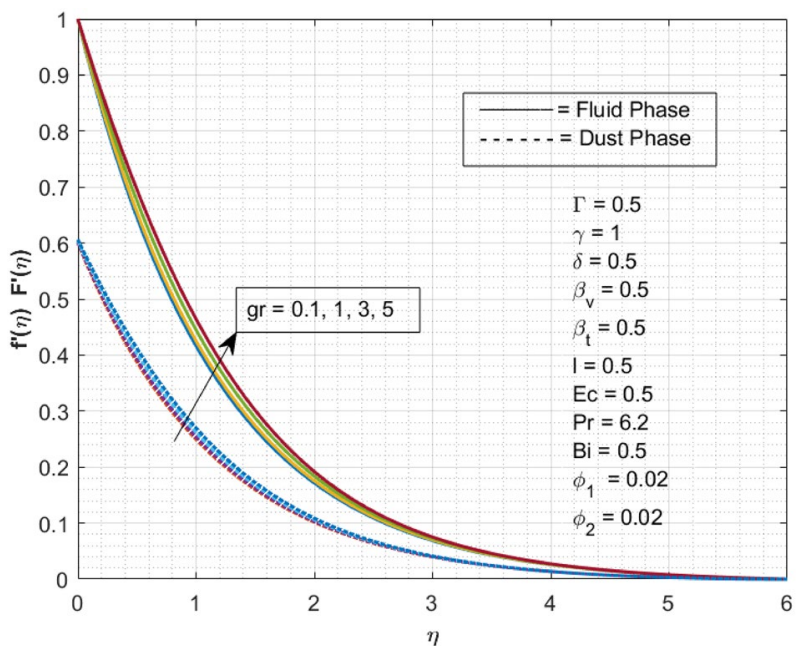

Fig. 3 Power of $g r$ over $f^{\prime}(\eta), F^{\prime}(\eta)$

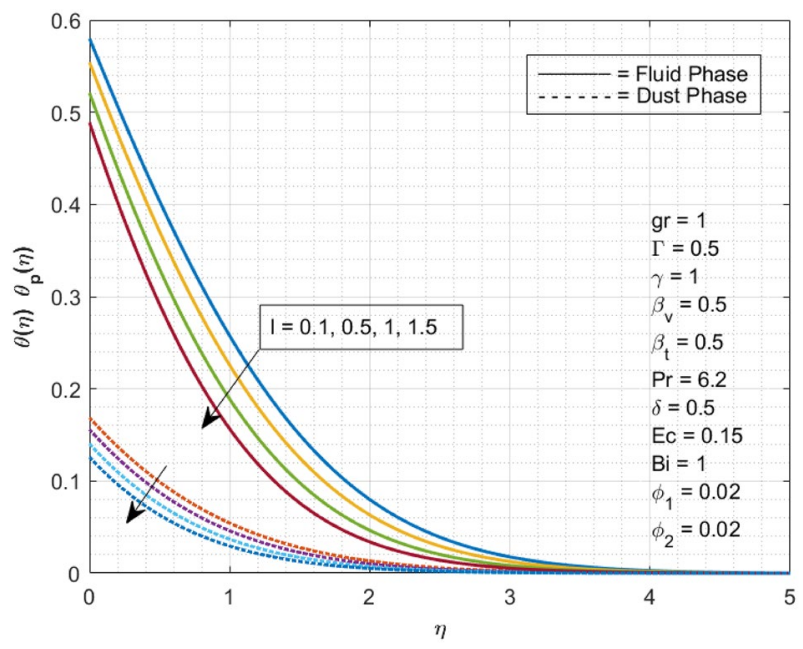

Fig. 4 Power of $/$ over $\theta(\eta), \theta_{p}(\eta)$

a display in Fig. 2. It is very clear from Fig. 2 that the liquid velocity is just as dust stage comparing to $(\delta=0.2)$ is least and the expansion of $(\delta)$ is to expand the velocity inside the boundary layer. Also $(\delta=0)$, the issue diminishes to flat surface. Henceforth, the speed inside the boundary layer on account of cylinder is bigger than the flat surface. It implies that the expanding in the cylinder measurement prompts the increasing in the velocity inside the boundary layer. The inclination in velocity gradient is faster in dust phase when compared to fluid phase. Figure 3 portrays the sway of $g r$ on velocity gradient. The rise in values of gr gradually heightens the velocity of both the phases. Enhance in mixed convection parameter causes high difference in environmental and surface temperature. This difference causes increment in thermal gradient which

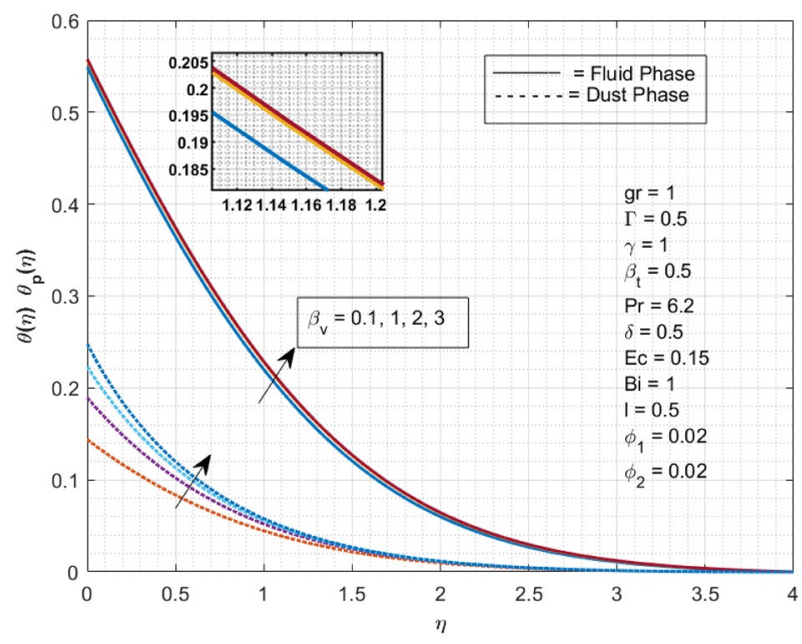

Fig. 5 Power of $\beta_{v}$ over $\theta(\eta), \theta_{p}(\eta)$

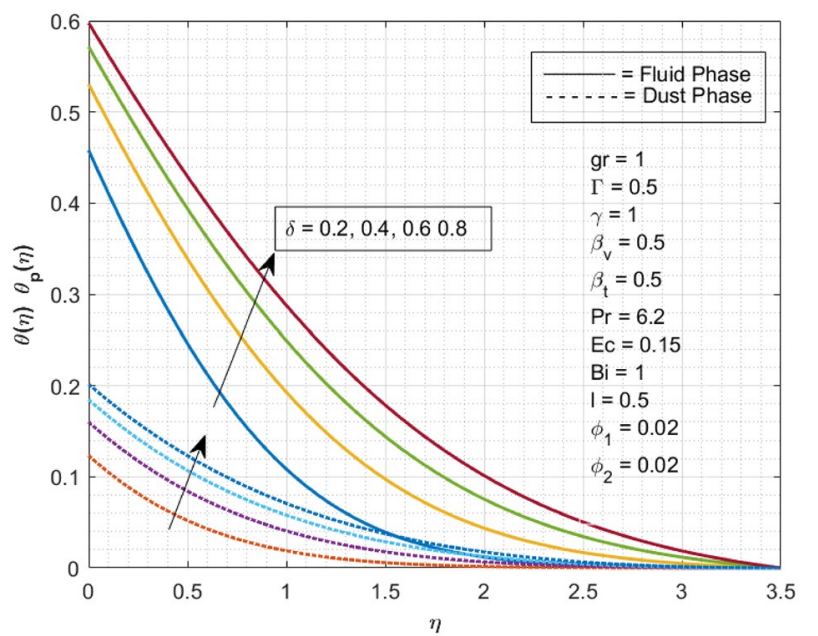

Fig. 6 Power of $\delta$ over $\theta(\eta), \theta_{p}(\eta)$

results in upshot of velocity in both the phases. The domination of mass concentration of the particle on thermal gradient of both phases is depicted in Fig. 4. One can detect from the figure that upsurge in I deteriorates the thermal profile. It is caused due to the dust particle mass concentration present in the liquid. Physically, it means that increasing value of $I$ enhance the number density of dust particles and decelerate velocity of the dusty liquid and thermal gradient.

Figure 5 represents the encouragement of $\beta_{v}$ on thermal gradient. It is detected from figure that upshot in $\beta_{v}$ gradually boosts up the thermal profile. The dust phase boundary layer thickness is fewer than the thickness related with liquid phase as shown in Fig. 5. The sway of $\delta$ on thermal gradient is portrayed in Fig. 6. It is obvious from plotted figure that, upshot in curvature parameter progresses the thermal profile of both phases. Figure 7 shows the sway of $E c$ on thermal 


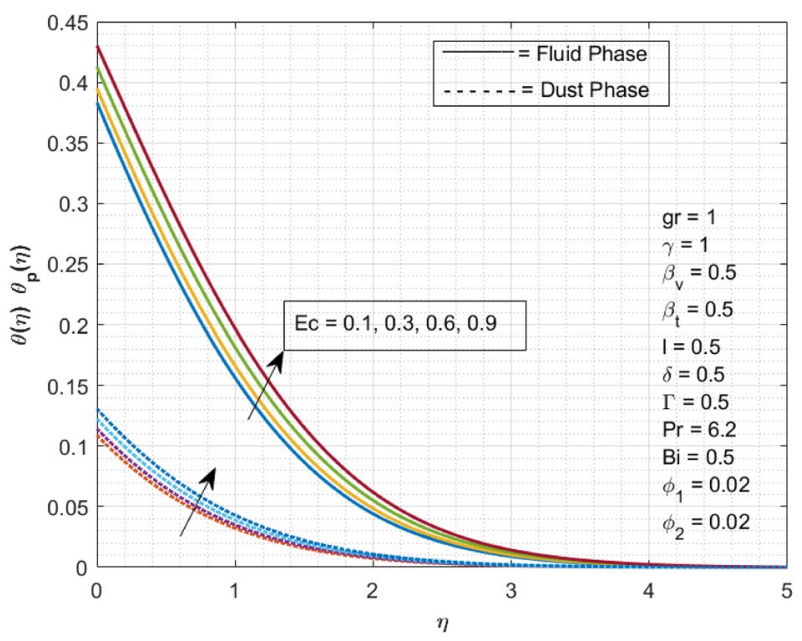

Fig. 7 Power of $E c$ over $\theta(\eta), \theta_{p}(\eta)$

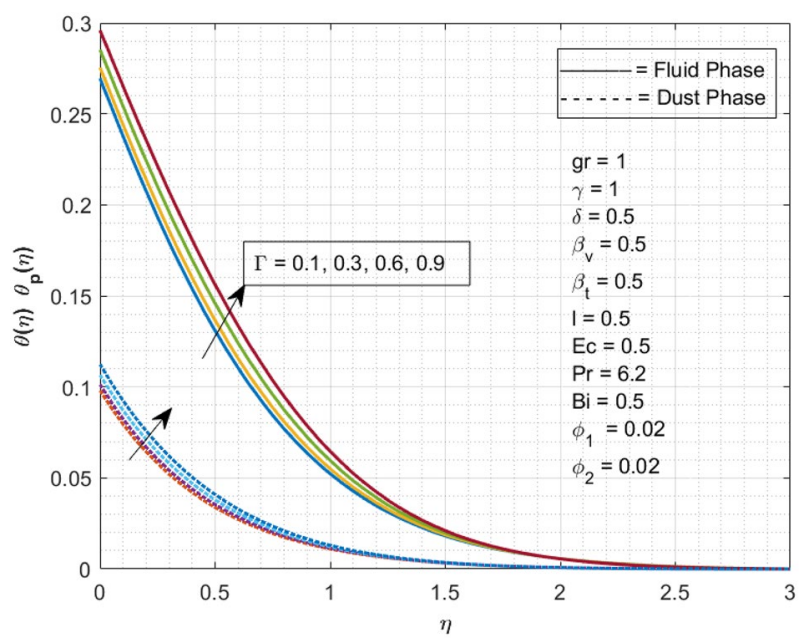

Fig. 8 Power of $\Gamma$ over $\theta(\eta), \theta_{p}(\eta)$

profile. It is observed from the plotted figure that heightening in $E c$ heightens the thermal gradient. It is obvious that, upsurge in Ec improve the inter-molecular motion and kinetic energy in fluid. As a result, this inter-molecular tension upsurges the thermal gradient of both the phases and of the related boundary layer. The impact of $\Gamma$ on thermal profile for both phases are displayed in Fig. 8. The heightening of $\Gamma$ enhances the thermal profile of both phases as displayed in Fig. 8. The fluid phase boundary layer thickness is greater than the thickness related with dust phase. Physically, the existence of heat generation effects has the ability to enhance the thermal state of the liquid which reasons for an upsurge in its temperature and hence fluid phase is more thermal than dust phase. The sway of $B i$ on thermal profile for both the phases are displayed in Fig. 9. The measure of

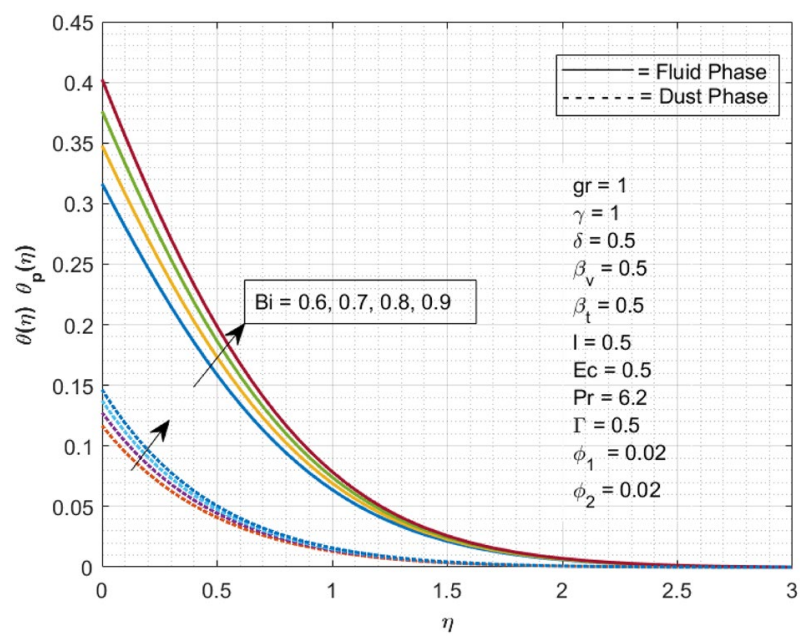

Fig. 9 Power of $B i$ over $\theta(\eta), \theta_{p}(\eta)$

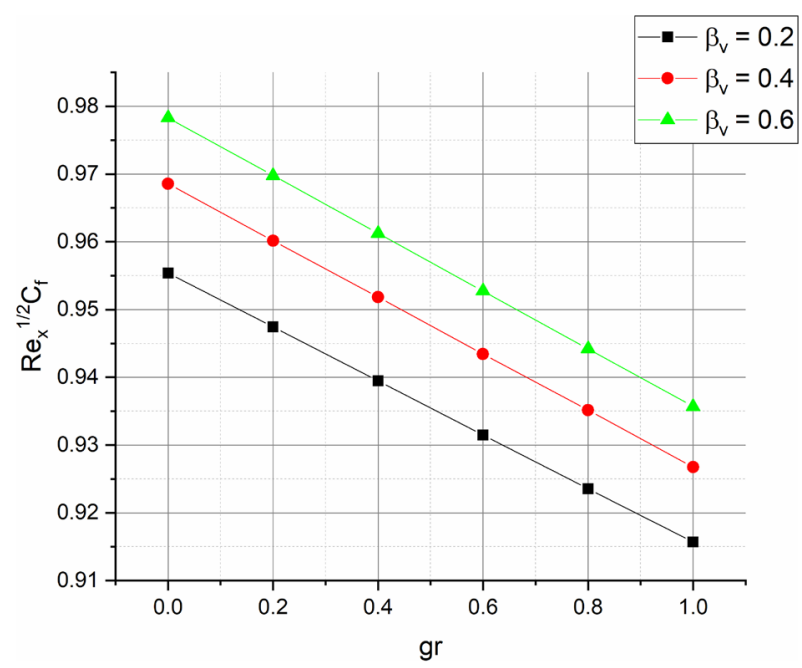

Fig. 10 Power of $\beta_{v}$ on $\operatorname{Re}_{x}^{1 / 2} C_{f}$

the interaction among convection at its surface and conduction in the solid is called as Biot number. Here, it is essential to mention that, the extreme values of $B i$ enhances the thermal profile of both the phases. Here, the Bi comprises the heat transfer coefficient and which increases for higher values of $B i$. Physically, inclination in Bi produces large heat transfer through convection which results in inclination of thermal field. Effect of $\beta_{v}$ on $R e_{x}{ }^{1 / 2} C_{f}$ is represented in Fig. 10. It is seen from figure that rise in values of $\beta_{v}$ enhances the $R e_{x}{ }^{1 / 2} C_{f}$. Figure 11 portrays the influence of $/$ on $R e_{x}{ }^{1 / 2} C_{f}$. One can observe from figure that rise in values of $I$ enhances the $\operatorname{Re}_{x}{ }^{1 / 2} C_{f}$. Further, it is evident from both the figures that $R e_{X}{ }^{1 / 2} C_{f}$ acts as a declining function of mixed convection parameter and growing function of $I$ and $\beta_{v}$. 


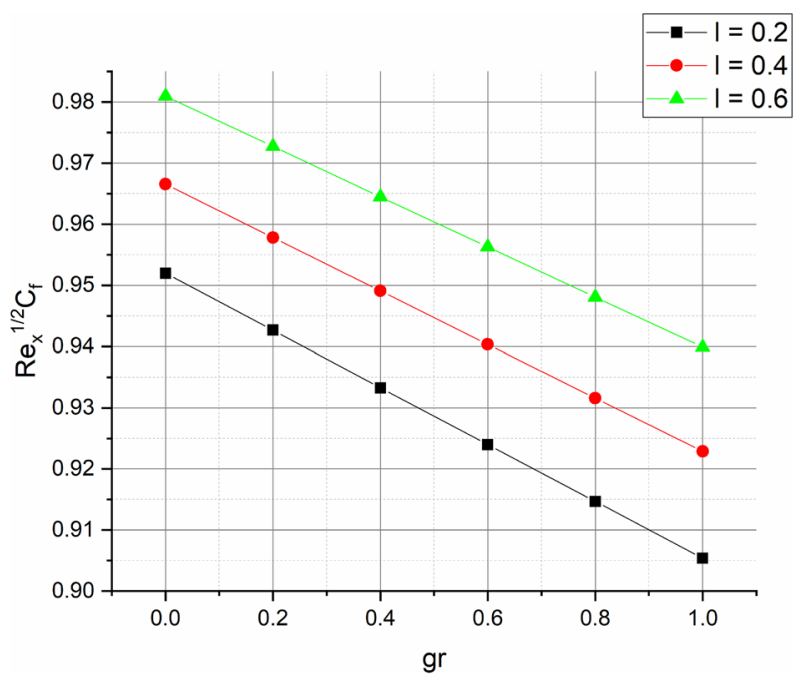

Fig. 11 Power of $/$ on $R e_{x}^{1 / 2} C_{f}$
Funding The authors have no affiliation with any organization with a direct or indirect financial interest in the topic talked about in the manuscript.

\section{Compliance with ethical standards}

Conflict of interest The authors have no conflicts of interest to announce. All co-authors have seen and agree with the contents of the manuscript and there is no financial interest to report.

Open Access This article is licensed under a Creative Commons Attribution 4.0 International License, which permits use, sharing, adaptation, distribution and reproduction in any medium or format, as long as you give appropriate credit to the original author(s) and the source, provide a link to the Creative Commons licence, and indicate if changes were made. The images or other third party material in this article are included in the article's Creative Commons licence, unless indicated otherwise in a credit line to the material. If material is not included in the article's Creative Commons licence and your intended use is not permitted by statutory regulation or exceeds the permitted use, you will need to obtain permission directly from the copyright holder. To view a copy of this licence, visit http://creativecommons .org/licenses/by/4.0/.

\section{References}

1. Hayat T, Qayyum S, Alsaedi A, Shafiq A (2016) Inclined magnetic field and heat source/sink aspects in flow of nanofluid with nonlinear thermal radiation. Int J Heat Mass Transf 103:99-107. https://doi.org/10.1016/j.ijheatmasstrans fer.2016.06.055

2. Khan JA, Mustafa M, Hayat T, Turkyilmazoglu M, Alsaedi A (2017) Numerical study of nanofluid flow and heat transfer over a rotating disk using Buongiorno's model. Int J Numer Methods Heat Fluid Flow 27(1):221-234. https://doi. org/10.1108/HFF-08-2015-0328

3. Kuttan BA, Manjunatha S, Jayanthi S, Gireesha BJ (2020) Performance of four different nanoparticles in boundary layer flow over a stretching sheet in porous medium driven by buoyancy force. Int J Appl Mech Eng 25(2):1-10. https://doi. org/10.2478/ijame-2020-0016

4. Vinita V, Poply V (2020) Impact of outer velocity MHD slip flow and heat transfer of nanofluid past a stretching cylinder. Mater Today Proc 26:3429-3435. https://doi.org/10.1016/j. matpr.2019.11.304

5. Mondal H, Das S, Kundu PK (2020) Influence of an inclined stretching cylinder over MHD mixed convective nanofluid flow due to chemical reaction and viscous dissipation. Heat Transf 49(4):2183-2193. https://doi.org/10.1002/htj.21714

6. Khan MI, Ahmad Khan S, Hayat T, Waqas M, Alsaedi A (2019) Modeling and numerical simulation for flow of hybrid nanofluid $(\mathrm{SiO} 2 / \mathrm{C} 3 \mathrm{H} 8 \mathrm{O} 2)$ and $(\mathrm{MoS} 2 / \mathrm{C} 3 \mathrm{H} 8 \mathrm{O} 2)$ with entropy optimization and variable viscosity. Int J Numer Methods Heat Fluid Flow 30(8):3939-3955. https://doi.org/10.1108/HFF-10-2019-0756

7. Farooq S, ljaz Khan M, Waqas M, Hayat T, Alsaedi A (2020) Transport of hybrid type nanomaterials in peristaltic activity of viscous fluid considering nonlinear radiation, entropy optimization and slip effects. Comput Methods Progr Biomed 184:105086. https://doi.org/10.1016/j.cmpb.2019.105086

8. Manjunatha S, Ammani Kuttan B, Ramesh GK, Gireesha BJ, Aly EH (2020) 3D flow and heat transfer of micropolar fluid suspended with mixture of nanoparticles $(\mathrm{Ag}-\mathrm{CuO} / \mathrm{H} 2 \mathrm{O})$ driven by an 
exponentially stretching surface. Multidiscip Model Mater Struct 16(6):1691-1707. https://doi.org/10.1108/MMMS-12-2019-0226

9. Abbas N, Nadeem S, Saleem A, Malik MY, Issakhov A, Alharbi FM (2021) Models base study of inclined MHD of hybrid nanofluid flow over nonlinear stretching cylinder. Chin J Phys 69:109-117. https://doi.org/10.1016/j.cjph.2020.11.019

10. Turkyilmazoglu M (2017) Magnetohydrodynamic twophase dusty fluid flow and heat model over deforming isothermal surfaces. Phys Fluids 29(1):013302. https://doi. org/10.1063/1.4965926

11. Manjunatha PT, Gireesha BJ, Prasannakumara BC (2017) Effect of radiation on flow and heat transfer of MHD dusty fluid over a stretching cylinder embedded in a porous medium in presence of heat source. Int J Appl Comput Math 3(1):293-310. https://doi.org/10.1007/s40819-015-0107-x

12. Gnaneswara Reddy M, Sudha Rani MVVNL, Ganesh Kumar K, Prasannakumar BC, Lokesh HJ (2020) Hybrid dusty fluid flow through a Cattaneo-Christov heat flux model. Phys Stat Mech Appl 551:123975. https://doi.org/10.1016/j.physa.2019.123975

13. Radhika M, Gowda RJP, Naveenkumar R, Siddabasappa BCP (2020) Heat transfer in dusty fluid with suspended hybrid nanoparticles over a melting surface. Heat Transf. https://doi. org/10.1002/htj.21972

14. Mallikarjuna HB, Nirmala T, Gowda RJP, Manghat R, Kumar RSV (2021) Two-dimensional Darcy-Forchheimer flow of a dusty hybrid nanofluid over a stretching sheet with viscous dissipation. Heat Transf. https://doi.org/10.1002/htj.22058

15. Abdelmalek Z, Nawaz M, Elmasry Y (2020) Simultaneous impact of hybrid nano and dust particles on enhancement of heat transfer in fluid with micro-rotation and thermal memory effects. Int Commun Heat Mass Transf 118:104871. https://doi. org/10.1016/j.icheatmasstransfer.2020.104871

16. Hayat T, Qayyum S, Alsaedi A, Asghar S (2017) Radiation effects on the mixed convection flow induced by an inclined stretching cylinder with non-uniform heat source/sink. PLoS ONE 12(4):e0175584. https://doi.org/10.1371/journal.pone.0175584

17. Khan MI, Tamoor M, Hayat T, Alsaedi A (2017) MHD boundary layer thermal slip flow by nonlinearly stretching cylinder with suction/blowing and radiation. Results Phys 7:1207-1211. https ://doi.org/10.1016/j.rinp.2017.03.009

18. Waqas $M, N a z S$, Hayat T, Alsaedi A (2019) Numerical simulation for activation energy impact in Darcy-Forchheimer nanofluid flow by impermeable cylinder with thermal radiation. Appl Nanosci 9(5):1173-1182. https://doi.org/10.1007/s13204-01800940-z

19. Mishra A, Kumar M (2020) Velocity and thermal slip effects on MHD nanofluid flow past a stretching cylinder with viscous dissipation and Joule heating. SN Appl Sci 2(8):1350. https://doi. org/10.1007/s42452-020-3156-7

20. Qayyum S, Hayat T, Shehzad SA, Alsaedi A (2018) Mixed convection and heat generation/absorption aspects in MHD flow of tangent-hyperbolic nanoliquid with Newtonian heat/ mass transfer. Radiat Phys Chem 144:396-404. https://doi. org/10.1016/j.radphyschem.2017.10.002

21. Alsaedi A, Hayat T, Qayyum S, Yaqoob R (2020) Eyring-Powell nanofluid flow with nonlinear mixed convection: Entropy generation minimization. Comput Methods Progr Biomed 186:105183. https://doi.org/10.1016/j.cmpb.2019.105183

22. Akbar Y, Abbasi FM, Shehzad SA (2020) Thermodynamical analysis for mixed convective peristaltic motion of silver-water nanoliquid having temperature dependent electrical conductivity. Appl Nanosci. https://doi.org/10.1007/s13204-020-01569-7

23. Ramesh GK, Manjunatha S, Roopa GS, Chamkha AJ (2020) Hybrid (ND-Co3O4/EG) nanoliquid through a permeable cylinder under homogeneous-heterogeneous reactions and slip effects. J Therm Anal Calorim. https://doi.org/10.1007/s1097 3-020-10106-1

24. Salahuddin T, Malik MY, Hussain A, Bilal S, Awais M (2016) MHD flow of Cattanneo-Christov heat flux model for Williamson fluid over a stretching sheet with variable thickness: using numerical approach. J Magn Magn Mater 401:991-997. https://doi. org/10.1016/j.jmmm.2015.11.022

25. Ijaz Khan M, Nigar M, Hayat T, Alsaedi A (2020) On the numerical simulation of stagnation point flow of non-Newtonian fluid (Carreau fluid) with Cattaneo-Christov heat flux. Comput Methods Progr Biomed 187:105221. https://doi.org/10.1016/j. cmpb.2019.105221

26. Hussain A, Malik MY, Khan M, Salahuddin T (2019) Application of generalized Fourier heat conduction law on MHD viscoinelastic fluid flow over stretching surface. Int J Numer Methods Heat Fluid Flow 30(6):3481-3496. https://doi.org/10.1108/ HFF-02-2019-0161

27. Ahmed A, Khan M, Ahmed J, Nadeem S (2020) Mixed convection in unsteady stagnation point flow of maxwell fluid subject to modified Fourier's Law. Arab J Sci Eng 45(11):9439-9447. https ://doi.org/10.1007/s13369-020-04724-y

28. Khan M, Ahmed A, Irfan M, Ahmed J (2020) Analysis of CattaneoChristov theory for unsteady flow of Maxwell fluid over stretching cylinder. J Therm Anal Calorim. https://doi.org/10.1007/ s10973-020-09343-1

29. Grubka LJ, Bobba KM (1985) Heat transfer characteristics of a continuous stretching surface with variable temperature. ASME J Heat Transf 107(1):248-250

30. Abel MS, Mahesha N (2008) Heat transfer in MHD viscoelastic fluid flow over a stretching sheet with variable thermal conductivity, non-uniform heat source and radiation. Appl Math Model 32(10):1965-1983

31. Ali ME (1994) Heat transfer characteristics of a continuous stretching surface. Wärme- Stoffübertrag 29(4):227-234

32. Ishak A, Nazar R, Pop I (2008) Hydromagnetic flow and heat transfer adjacent to a stretching vertical sheet. Heat Mass Transf 44(8):921

33. Shafie S, Gul A, Khan I (2016) Molybdenum disulfide nanoparticles suspended in water based nanofluids with mixed convection and flow inside a channel filled with saturated porous medium. AIP Conf Proc 1775(1):030042

Publisher's Note Springer Nature remains neutral with regard to jurisdictional claims in published maps and institutional affiliations. 